\title{
Design of Serially Concatenated Continuous Phase Modulation with Symbol-Wise Interleaving
}

\author{
Jun Ning, Member, IEEE, and Minyue Fu, Fellow, IEEE
}

\begin{abstract}
In this letter we consider serially concatenated continuous phase modulation (SCCPM) schemes with symbolwise interleaving and an additional intra-symbol interleaving. We use extrinsic information transfer (EXIT) chart to optimize the outer code and intra-symbol interleaver. We show that, for various continuous phase modulation (CPM) parameters, the outer code with memory 2 may not always be the best choice. For CPM with modulation index $h=1 / 2$, considerable improvements can be achieved by moderately increasing the outer code memory.
\end{abstract}

Index Terms - continuous phase modulation, serially concatenated CPM, symbol-wise interleaving, EXIT chart.

\section{INTRODUCTION}

C ONTINUOUS phase modulation (CPM) plays an important role in radio and satellite communications due to its constant envelope property and high spectral efficiency [1], [2]. The spectral efficiency of a CPM scheme depends on a number of parameters. These include the shape function $g(t)$ (rectangular, raised cosine or Gaussian), the duration $L$ of the shape function, the symbol period $T$, the cardinality of the CPM symbols $M$, and the modulation index $h$. In this letter, the modulation index is restricted to the form of $h=1 / P$, where $P$ is the number of phase states. A high spectral efficiency can be achieved by increasing the values of $L, M$ and $P$. This comes with the tradeoff of decoding complexity and bit-error-rate (BER) performance. On the other hand, decoding complexity and BER performance can be improved by increasing the modulation index $h$. To improve the power efficiency by coding, a CPM system is typically cascaded with an outer code. Shortly after the invention of turbo codes, serially concatenated CPM (SCCPM) showed their advantages in power efficiency [3]-[12].

A typical SCCPM system consists of a binary outer code, a bit-wise interleaver and an inner code (CPM) which is connected to the channel directly. When $M>2$, a mapper is also needed to bridge between the bit-wise interleaver and the non-binary CPM. Iterative methods are typically used for decoding. Recently, it is shown that SCCPM systems with symbol-wise interleaving can achieve substantial improvement in BER performance over their counterparts with bit-wise interleaving [11], [12]. The outer code in a symbol-wise interleaved SCCPM can still be a binary code, provided that the symbols for the outer code have a size of alphabet equal to $M$. For an SCCPM, nonrecursive convolutional codes (CCs)

Manuscript received October 15, 2008. The associate editor coordinating the review of this letter and approving it for publication was S. Buzzi.

The authors are with the School of Electrical Engineering and Computer Science, University of Newcastle, Callaghan, NSW 2308 Australia (e-mail: \{jun.ning, minyue.fu\}@newcastle.edu.au).

Digital Object Identifier 10.1109/LCOMM.2009.081690

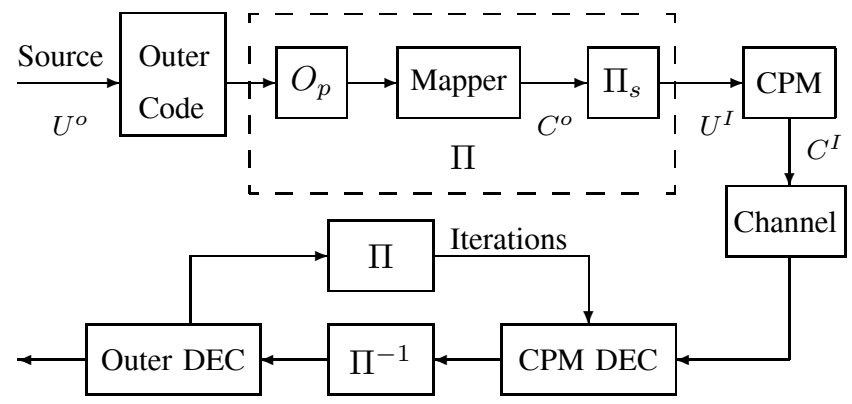

Fig. 1. The proposed symbol-wise interleaved SCCPM scheme.

with very low memory, acting as outer codes, have shown their advantages over those with high memory. Typically, nonrecursive convolutional code with the generator matrix $G(D)=\left[1+D+D^{2}, 1+D^{2}\right]$, where $D$ is a delay operator, or $(7,5)$ in octal form is widely-used and believed to offer excellent performance in concatenation with a large class of CPMs. To the authors' knowledge, there is no literature showing the advantage of using outer code with memory higher than 2 .

In this letter we consider symbol-wise interleaved SCCPM schemes. Motivated by the approach in [13], we add an intrasymbol interleaver in the schemes to further improve the BER performance. For the proposed schemes we use extrinsic information transfer (EXIT) chart technique to optimize the outer code and intra-symbol interleaver. For the SCCPM schemes with modulation index $h=1 / 2$, we show that considerable improvements can be achieved by moderately increasing the outer code memory.

\section{The Proposed SCCPM}

The proposed scheme shown in Fig. 1 is similar to the symbol-wise interleaved scheme in [11] except for the addition of an intra-symbol interleaver $O_{p}$. The motivation of adding an intra-symbol interleaver is based on the idea presented in [13] as well as the observation that the ordering of the output bits within a coded symbol from the outer encoder has some impact on the BER performance. The choices for $O_{p}$ can be fixed or periodic. Take the outer encoder $(7,5)$ as an example, intra-symbol interleaver $O_{p}=\left[\begin{array}{ll}2 & 1\end{array}\right]$ indicates that the order of output bits from the two branches of encoder $(7,5)$ is fixed and the bit from branch 5 precedes the bit from branch 7 within the output symbol. Another example is $O_{p}=\left\{\left[\begin{array}{ll}1 & 2\end{array}\right],\left[\begin{array}{ll}2 & 1\end{array}\right]\right\}$ which means that $O_{p}$ is chosen to be $\left[\begin{array}{ll}2 & 1\end{array}\right]$ and $\left[\begin{array}{ll}1 & 2\end{array}\right]$ periodically.

In Fig. 1 an information binary sequence $U^{o}$ is fed into the outer encoder. For each input symbol which consists of 

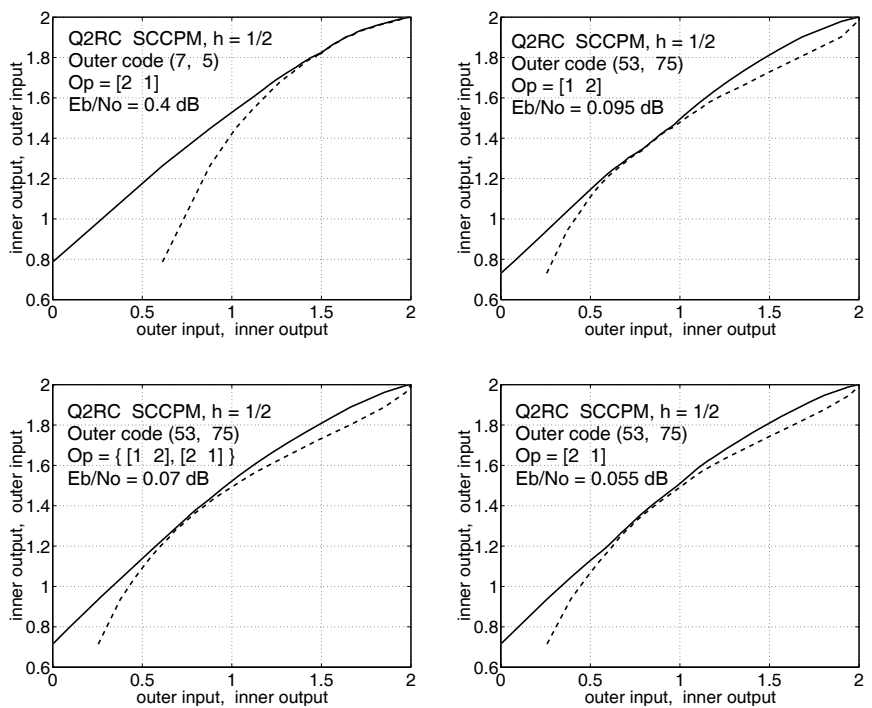

Fig. 2. EXIT charts of the symbol-wise interleaved SCCPMs.

$n_{i}$ bits at each time instant, the encoder generates a coded symbol consisting of $n_{o}$ bits. The intra-symbol interleaver $O_{p}$ permutates the order of the $n_{o}$ bits within the coded symbol and the mapper converts each symbol of $O_{p}$ into a symbol according to Natural Binary Code (NBC) or Gray mapping rule, assuming that $n_{o}=\log _{2} M$. The symbol sequence $C^{o}$ from the mapper is then interleaved by the symbol-wise interleaver $\Pi_{s}$ into another symbol sequence $U^{I}$ which is treated as the input of CPM. Through CPM, the sequence $U^{I}$ is modulated to give the CPM symbol sequence $C^{I}$ before it is transmitted over the communication channel. In this paper, symbol-wise interleavers are chosen to be S-random interleavers [14].

\section{Outer Code Design Using EXIT Chart}

EXIT chart technique is a well-known numerical tool for analyzing the convergence behavior of iterative decoders and predicting the convergence threshold of turbo-like concatenated codes. The convergence threshold is the lowest signalto-noise ratio at which the decoding can converge at a given BER. In this letter, we restrict ourselves to quaternary CPMs with raised cosine shape function of duration 2 and 3, denoted by Q2RC CPM and Q3RC CPM, respectively. For the purpose of optimization, we choose the outer codes to be different convolutional codes (CCs) with memory varying from 2 to 5. Also, we try different intra-symbol interleavers for the schemes. For comparison, we choose the modulation index $h$ to be $1 / 2$ and $1 / 4$ and use both bit-wise interleaving and symbol-wise interleaving. EXIT charts are computed using the approach in [11] which is based on [15], [16].

From the EXIT charts, we have the following observations. Gray mapping is always preferred to NBC mapping for the bitwise interleaved schemes, whereas NBC mapping performs better for the most symbol-wise interleaved schemes. For bit-wise interleaved schemes and symbol-wise interleaved schemes with $h=1 / 4$, outer code $(7,5)$ are superior to those with higher memory. Nevertheless, for symbol-wise interleaved schemes with $h=1 / 2$, outer code $(7,5)$ is

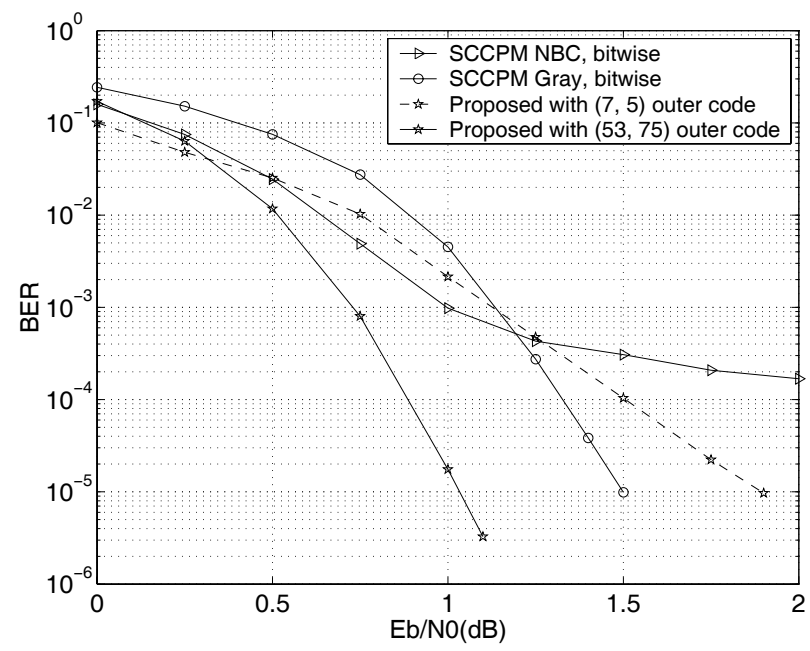

Fig. 3. Simulation results of the coded quaternary 2 RC CPMs with rate-1/2 binary outer code, $h=1 / 2, N=1024$ and 12 decoding iterations.

not the best choice and improvements can be achieved by increasing code memory moderately. In addition, a symbolwise interleaved scheme with the optimized outer code, mapping and intra-symbol interleaver substantially outperforms its bit-wise interleaved counterparts. The best intra-symbol interleaver varies for different schemes.

Fig. 2 shows the EXIT charts of symbol-wise interleaved SCCPMs with Q2RC CPM and $h=1 / 2$ as typical examples. The input information block has 20,000 bits. The EXIT chart on the left-top of Fig. 2 shows the decoding trajectories and the convergence threshold in $E_{b} / N_{0}$ of the scheme using CC $(7,5)$ and $O_{p}=[21]$. Other EXIT charts in the figure are from the schemes using CC $(53,75)$, with difference in $O_{p}$. We observe that each pair of EXIT curves associated with CC $(53,75)$ match much better than those associated with $\mathrm{CC}$ $(7,5)$. In particular, the schemes with $\mathrm{CC}(53,75)$ and $O_{p}=$ $[21]$ gives the lowest convergence threshold, although different intra-symbol interleavers do not cause much difference.

\section{Simulations ANd COMParisons}

In this section, we present and compare some simulation results when AWGN channels and coherent demodulations are assumed. The input block size for the outer codes is $N=1024$ bits and the outer code rate is $1 / 2$. For each comparison, we keep the CPM parameters unchanged. Also for the convenience of comparison, we only show the simulation results of symbol-wise interleaved schemes with NBC mapping in the following figures.

Fig. 3 shows the simulated results for SCCPMs with Q2RC CPM and $h=1 / 2$ using bit-wise or symbol-wise interleaving. When the outer code is CC $(7,5)$, the result for the symbolwise interleaved scheme is worse than that of its bit-wise counterpart. However, when the outer code is CC $(53,75)$, a gain of about $0.5 \mathrm{~dB}$ at BER of $10^{-5}$ is observed for the symbol-wise interleaved scheme over the best bit-wise interleaved scheme.

To show the impact of an intra-symbol interleaving, Fig. 4 compares the simulated results of the symbol-wise interleaved 


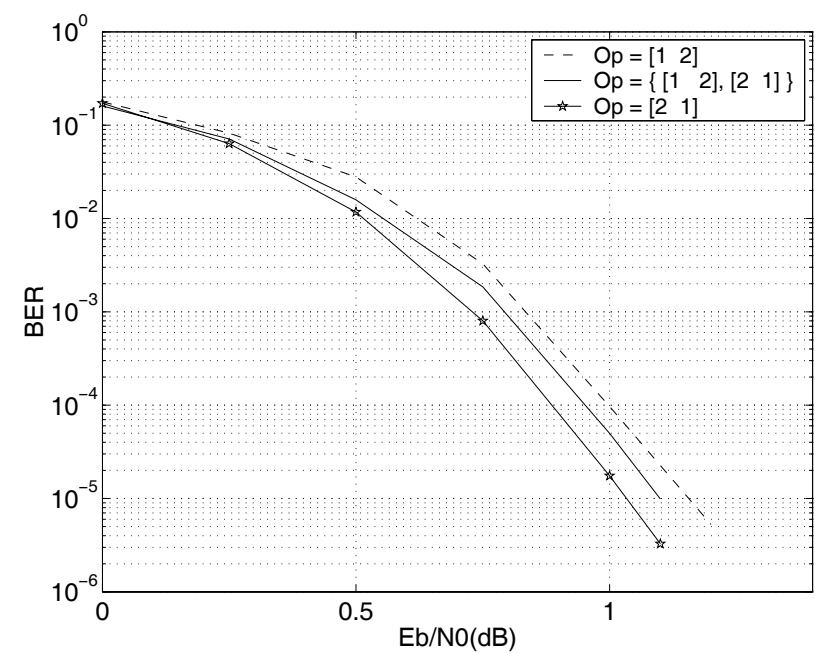

Fig. 4. Simulation results of the coded quaternary $2 \mathrm{RC}$ CPMs with rate$1 / 2$ binary outer code $(53,75)$, various intra-symbol interleavers, $h=1 / 2$, $N=1024$ and 12 decoding iterations.

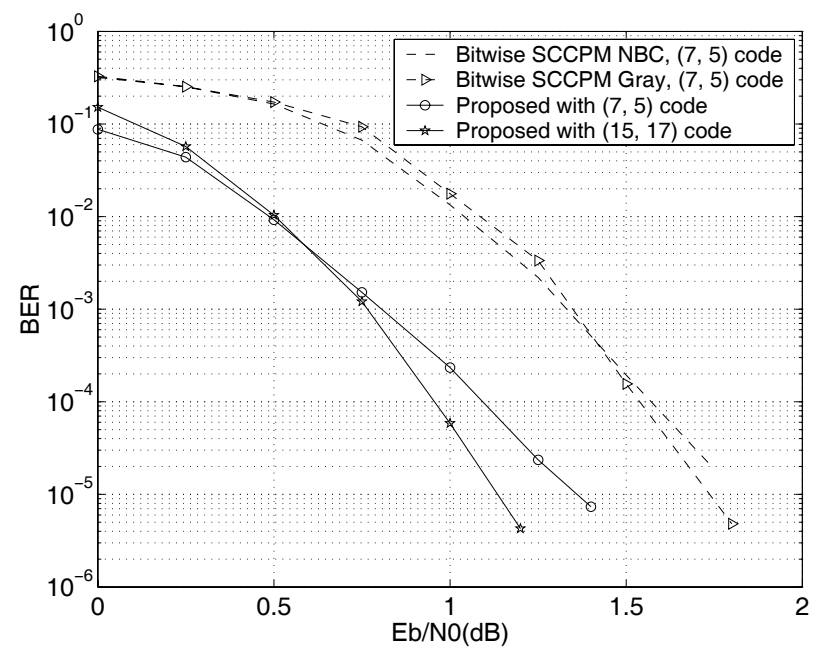

Fig. 5. Simulation results of the coded quaternary 3RC CPMs with rate- $1 / 2$ binary outer code, $h=1 / 2, N=1024$ and 12 decoding iterations.

Q2RC CPM schemes with outer code $(53,75)$ but various intra-symbol interleavers. It turns out that intra-symbol interleaver $O_{p}=[21]$ gives the best performance while $O_{p}=\left[\begin{array}{ll}1 & 2\end{array}\right]$ performs worst, although the difference between them is only about $0.12 \mathrm{~dB}$ at BER of $10^{-5}$.

For SCCPMs with Q3RC CPM, Fig. 5 shows the simulated results for SCCPMs with Q3RC CPM and $h=1 / 2$ using bit-wise or symbol-wise interleaving. We observe that the symbol-wise interleaved schemes outperform the bit-wise interleaved schemes with $(7,5)$ outer code. Using symbolwise interleaving, the schemes with $(15,17)$ outer code and $O_{p}=\left\{\left[\begin{array}{ll}1 & 2\end{array}\right],\left[\begin{array}{ll}2 & 1\end{array}\right]\right\}$ outperforms the schemes with $(7,5)$ outer code and $O_{p}=\left[\begin{array}{ll}2 & 1\end{array}\right]$ by about $0.25 \mathrm{~dB}$.

From the simulation results of the SCCPM schemes with $h=1 / 2$ and Q2RC CPM or Q3RC CPM, we observed that considerable improvements can be achieved by moder- ately increasing the outer code memory. Note that for these examples, outer codes with memory higher than 2 do not give any improvement for bit-wise interleaved schemes. These simulated results are in line with our EXIT chart analysis.

\section{CONCLUSion}

In this letter we have considered symbol-wise interleaved SCCPM schemes and designed outer codes using EXIT chart. It turned out that, for the schemes with moderate modulation index, the outer code with memory 2 is superior, while for the schemes with high modulation index, the outer codes with moderate memory are preferred. We have also shown that the use of an additional intra-symbol interleaving can help the SCCPM systems achieve the best decoding performance. Finally, we have presented some simulation results to show the superior performance of the proposed schemes.

\section{REFERENCES}

[1] J. Anderson, T. Aulin, and C. Sundberg, Digital Phase Modulation. New York: Plenum Press, 1986.

[2] J. G. Proakis, Digital Communications, 3rd ed. McGraw-Hill, 1995.

[3] K. Narayanan and G. Stüber, "A serial concatenation approach to iterative demodulation and decoding," IEEE Trans. Commun., vol. 47, no. 7, pp. 956-961, July 1999.

[4] C. Brutel, J. Boutros, and F. Belvèze, "Serial encoding and iterative detection of continuous phase modulations," in Proc. IEEE Global Telecomm. Conf. GLOBECOM'99, 1999, pp. 2375-2379.

[5] R. Balasubramanian and M. P. Fitz, "Soft output detection of CPM signals in frequency flat, Rayleigh fading," IEEE J. Select. Areas Commun., vol. 18, no. 7, pp. 1145-1152, July 2000.

[6] K. Narayanan and G. Stüber, "Performance of trellis-coded CPM with iterative demodulation and decoding," IEEE Trans. Commun., vol. 49, no. 4, pp. 676-687, Apr. 2001.

[7] P. Moqvist and T.Aulin, "Serially concatnated continuous phase modulation with iterative decoding," IEEE Trans. Commun., vol. 49, no. 11, pp. 1901-1915, Nov. 2001.

[8] Y. Xu and E. Geraniotisz, "Iterative demodulation and decoding of highorder CPM signals in Rician fading channels," International J. Satellite Commun., vol. 20, pp. 363-378, 2002.

[9] X. Zhang and M. Fitz, "Soft output diversity combining for CPM signals over space-time correlated Rayleigh fading channels," in Proc. IEEE International Conference on Communications, ICC'01, vol. 2, June 2001, pp. 580-585.

[10] — , "Soft-output demodulator in space-time coded continuous phase modulation," IEEE Trans. Signal Processing, vol. 50, no. 10, pp. 2589 2598, Oct. 2002.

[11] M. Xiao and T. Aulin, "Serially concatenated continuous phase modulation with symbol interleavers: performance, properties and design principles," in Proc. IEEE Global Telecommunications Conference 2004 (GLOBECOM'04), vol. 1, Nov. 2004, pp. 179-183.

[12] _ - "Serially concatenated continuous phase modulation with convolutional codes over rings," IEEE Trans. Commun., vol. 54, pp. 1387-1396, Aug. 2006.

[13] C. Berrou, M. Jezequel, C. Douillard, and S. Kerouedan, "The advantages of non-binary turbo codes," in Proc. IEEE Information Theory Workshop 2001, Sept. 2001, pp. 61-63.

[14] S. Dolinar and D. Divsalar, "Weight distributions for turbo codes using random and nonrandom permutations," Jet Propulsion Lab., Pasadena, CA, USA, Tech. Rep. TDA Progress Rep. 42-122, Aug. 1995.

[15] S. Brink, "Convergence behavior of iteratively decoded parallel concatenated codes," IEEE Trans. Commun., vol. 49, no. 10, pp. 1727-1737, Oct. 2001.

[16] B. Scanavino, G. Montorsi, and S. Benedetto, "Convergence properties of iterative decoders working at bit and symbol level," in Proc. IEEE Global Telecommunications Conference 2001 (GLOBECOM'01), vol. 2, Nov. 2001, pp. 1037-1041. 